\title{
Immunohistochemical expression of mTOR negatively correlates with PTEN expression in gastric carcinoma
}

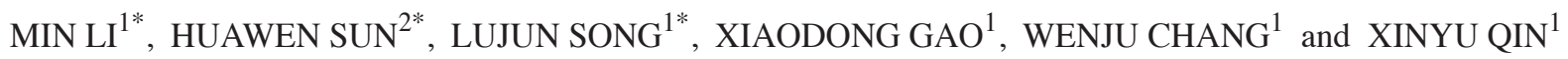 \\ ${ }^{1}$ Department of General Surgery, Zhongshan Hospital, Fudan University, Shanghai; \\ ${ }^{2}$ Department of Gastrointestinal Surgery, Renmin Hospital of Wuhan University, Wuhan, P.R. China
}

Received June 20, 2012; Accepted September 10, 2012

DOI: $10.3892 / \mathrm{ol} .2012 .930$

\begin{abstract}
The phosphoinositide-3 kinase (PI3K)-AKTmammalian target of rapamycin (mTOR) pathway is a cellular pathway involved in cell growth, tumorigenesis and cell invasion which is frequently activated in various types of cancer. The downstream effector of the pathway is mTOR which is important in cellular growth and homeostasis and aberrant activation of mTOR has been reported in several types of cancer. The tumor suppressor gene phosphatase and tensin homolog (PTEN) is essential in this pathway for inhibiting tumor invasion and metastasis. However, the involvement of mTOR and PTEN in the progression of human gastric cancer remains to be identified. Immunohistochemical staining was performed to detect the expression of mTOR and PTEN in paraffin-embedded gastric tissue sections obtained from 33 patients with gastric cancer and 30 normal controls. The expressed mTOR was mainly distributed in the cytoplasm, while PTEN was mainly localized to the nucleus. By considering negative mTOR expression with positive PTEN expression as one group and negative PTEN expression with positive mTOR expression as the other, significant statistical differences were observed in various categories, including histological types and metastatic and clinical pathology stages, between the 2 groups $(\mathrm{P}<0.01$ or 0.05$)$. The results indicated that the expression levels of mTOR and PTEN were negatively correlated in the PI3K-AKT-mTOR signaling pathway. Combined detection of mTOR and PTEN expression may be used to evaluate the degree of malignancy in gastric cancer and may be a useful marker for the early diagnosis of gastric cancer.
\end{abstract}

Correspondence to: Professor Xinyu Qin, Department of General Surgery, Zhongshan Hospital, Medical School, Fudan University, 180 Fenglin Road, Shanghai 200032, P.R. China

E-mail: qin.xinyu@zs-hospital.sh.cn

*Contributed equally

Key words: gastric carcinoma, mTOR, PTEN, immunohistochemistry

\section{Introduction}

Despite a declining incidence rate in the United States and a number of other Western countries, gastric cancer continues to be a worldwide health problem with more than 600,000 cases reported annually, far higher than pancreatic cancer with 125,000 cases (1). Gastric cancer is the most common gastrointestinal malignancy in East Asia, Eastern Europe and parts of Central and South America and is the second leading cause of cancer-related mortality (2). Despite improvements in surgery, radiotherapy and cytotoxic chemotherapy, survival rates for advanced gastric cancer are poor. Five years after multimodal treatment, less than $40 \%$ of Western patients with stage II or III disease are likely to be alive. At metastatic stage IV disease, the mean survival is only 10 months (3).

The phosphoinositide-3 kinase (PI3K)-AKT-mammalian target of rapamycin (mTOR) pathway is an important cellular pathway involved in cell growth, tumorigenesis, cell invasion and drug response $(4,5)$. PI $3 \mathrm{~K}$ is a heterodimer of 85 - and $110-\mathrm{kDa}$ subunits and has a tyrosine kinase activity, the activation of which stimulates the production of phosphatidylinositol 3,4,5-triphosphate resulting in activation of the kinases PDK1 and Akt. Akt is a kinase that phosphorylates a variety of target molecules to mediate signals, including mTOR, while mTOR phosphorylates and activates p70 S6 kinase (S6K)-1 and also inhibits eukaryotic translation initiation factor 4E-binding protein (4E-BP), resulting in enhanced protein synthesis and cell proliferation (6-8). This pathway is frequently activated in numerous types of cancer and uncontrolled PI3K-AKT-mTOR signaling may also result in a poor clinical outcome in lung, cervical, ovarian and esophageal cancers $(4,6)$.

mTOR was identified in 1994 by several groups of investigators as the kinase targeted by rapamycin linked to the cellular protein FKBP12 (FK506-binding protein) (9). It was therefore also named FKBP-RAP-associated protein (FRAP), RAP FKBP12 target (RAFT) 1 and RAP target (RAPT) 1. mTOR is a $289-\mathrm{kDa}$, ubiquitously expressed, evolutionarily conserved serine/threonine protein kinase (9) which is important in cellular protein synthesis and energy balance, affecting numerous aspects of cell growth and proliferation, including differentiation, cell-cycle progression, angiogenesis, protein degradation and apoptosis (10). mTOR is also instrumental in protein translation initiation (the rate-limiting step of protein synthesis) by enabling the recruitment of ribosomes to mRNA 
by eukaryotic initiation factor. Consequently, mTOR activates its downstream mediator ribosomal $\mathrm{S} 6 \mathrm{~K}$ and is responsible for the progression of the cell from G0/G1 to S phase (11). Consistent with its essential role in cell growth, aberrant activity of the mTOR pathway is frequently observed in a number of types of cancer (12).

Phosphatase and tensin homolog deleted on chromosome 10 (PTEN) is critical in cell growth, migration and death. It is mutated or deleted at a high frequency in various human cancer tissues to promote tumorigenesis (13). The PI3K-AKT-mTOR pathway is one of the most upregulated pathways in neoplastic cells through mechanisms such as PTEN loss of function or PI3K activating mutations (14). PTEN antagonizes PI3K pathways by dephosphorylating phosphatidylinositol 3,4,5-triphosphate to convert it back to phosphatidylinositol 4,5-biphosphate. Thus, PTEN is considered to be a negative regulator of the PI3K-AKT-mTOR pathway (15).

The aim of the present study was to explore the involvement of the PI3K-AKT-mTOR signaling pathway in the progression of human gastric cancer. The expression levels of mTOR and PTEN in human gastric cancer tissues were determined using immunohistochemical study using biopsies from 33 patients and correlations with pathological parameters and prognoses were evaluated.

\section{Materials and methods}

Patients and tumor samples. Paraffin-embedded sections were obtained from patients with gastric cancer who had undergone surgery at Renmin Hospital of Wuhan University (Wuhan, China) between 2005 and 2008. Tissues obtained from 30 cases of chronic superficial gastritis diagnosed by gastroscopic biopsy were used as control samples for the immunohistochemical staining. General informed consent with regard to the use of the patients' tissue specimens and clinical information was obtained from all patients. None of the patients recruited in this study had undergone chemotherapy or radiotherapy prior to surgery. The patients' clinicopathological data are summarized in Table I. The histological diagnosis was determined using hematoxylin and eosin staining according to the WHO criteria (16). Pathological staging was performed according to the American Joint Committee on Cancer (AJCC) Cancer Staging Manual revised in 2010 (17). This study was approved by the Institutional Ethics Board of the Renmin Hospital of Wuhan University.

Immunohistochemical staining. Formalin-fixed, paraffin-embedded tissue blocks obtained from human tissue were cut into $4-\mu \mathrm{m}$ thick sections and mounted on adhesive-coated glass slides. mTOR was detected with a rabbit monoclonal anti-mTOR antibody (Cell Signaling Technology, Inc., Danvers, MA, USA) and PTEN was observed with a mouse monoclonal anti-PTEN antibody (Maxim Biotechnology Development Co. Ltd, Fuzhou, China). Sections were dewaxed in xylene and rehydrated using graded ethanol and were then incubated in 3\% solution of hydrogen peroxide in methanol for $10 \mathrm{~min}$ to inactivate endogenous peroxidase. This was followed by an antigen retrieval step. The slides were placed in $0.01 \mathrm{~mol} / 1$ citrate
Table I. Clinicopathological parameters of the patients in the study.

\begin{tabular}{lr}
\hline Factors & Values \\
\hline Age, years, mean (range) & $51.6 \pm 11.6(24-74)$ \\
Gender, n (\%) & $15(45.5)$ \\
Male & $18(54.5)$ \\
Female & \\
Invasive depth, n (\%) & $6(18.2)$ \\
Early stage & $27(81.8)$ \\
Advanced stage & $15(45.5)$ \\
Differentiation, $\mathrm{n}(\%)$ & $18(54.5)$ \\
Well and moderate & \\
Poor & $16(48.5)$ \\
Lymph node metastasis, $\mathrm{n}(\%)$ & $17(51.5)$ \\
Positive & \\
Negative & $18(54.5)$ \\
Pathological stage, $\mathrm{n}(\%)$ & $15(45.5)$ \\
I+II &
\end{tabular}

buffer solution $(\mathrm{pH} 6.0)$ and heated $\left(92-100^{\circ} \mathrm{C}\right)$ for $10 \mathrm{~min}$ in a microwave oven. Following a wash with phosphate-buffered saline (PBS; pH 7.2) buffer, the sections were covered with $2 \%$ normal serum for $20 \mathrm{~min}$ at room temperature to eliminate non-specific binding of the antibody and were then incubated overnight at $4^{\circ} \mathrm{C}$ with the primary antibodies diluted in PBS. After washing with PBS, the secondary biotinylated antibody was added for a $20 \mathrm{~min}$ incubation at $37^{\circ} \mathrm{C}$. Slides were then rinsed with $\mathrm{PBS}$ and treated with streptavidin-peroxidase solution for $10 \mathrm{~min}$. Tissue sections were washed once in PBS buffer and covered with 3,3'-diaminobenzidine solution for $10 \mathrm{~min}$. Finally, the specimens were counterstained with hematoxylin. Normal gastric sections served as positive controls while negative control slides were incubated with the antibody diluents instead of the primary antibody.

Evaluation of slides. Immunohistochemical staining was evaluated by 3 independent experienced pathologists who were blinded to the clinicopathological parameters and clinical outcomes of the patients. In cases of disagreement between the observers slides were re-evaluated until a consensus was achieved. The sections were examined at x200 magnification using light microscopy. The immunostaining was considered to be positive when the neoplastic cells exhibited specific immunoreactivity in the cytoplasm for mTOR or in the nucleus for PTEN. The immunostaining results were assessed semiquantitatively. For each sample the positive rate was calculated according to the percentage of positive cells of all counted cells from 5 randomly selected representative fields. Additionally, the expression was classified according to the percentage of stained tumor cells as low expression $(-,<10 \%$ positive carcinoma cells), intermediate expression $(+, \geq 10 \%$ and $<50 \%$ positive carcinoma cells) and high expression $(++$, $\geq 50 \%$ positive carcinoma cells) (18). 

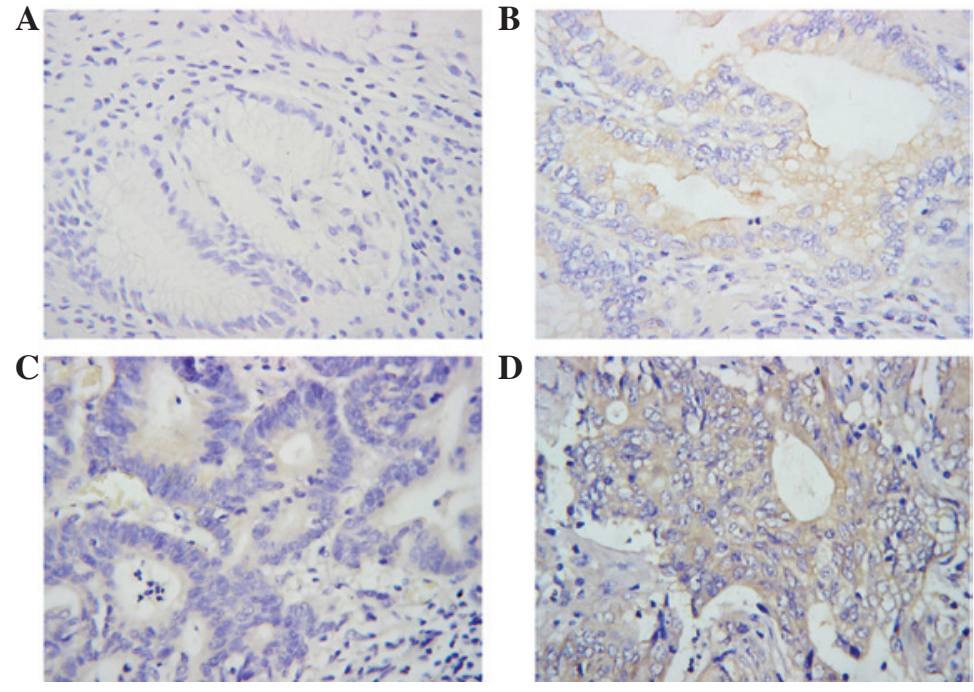

Figure 1. mTOR was mainly distributed in the cytoplasm. (A) Staining was hardly detected in normal gastric tissues and (B) was weaker in well-differentiated gastric cancer but was stronger in (C) moderately and (D) poorly differentiated tumors. Original magnification, x400. mTOR, mammalian target of rapamycin..
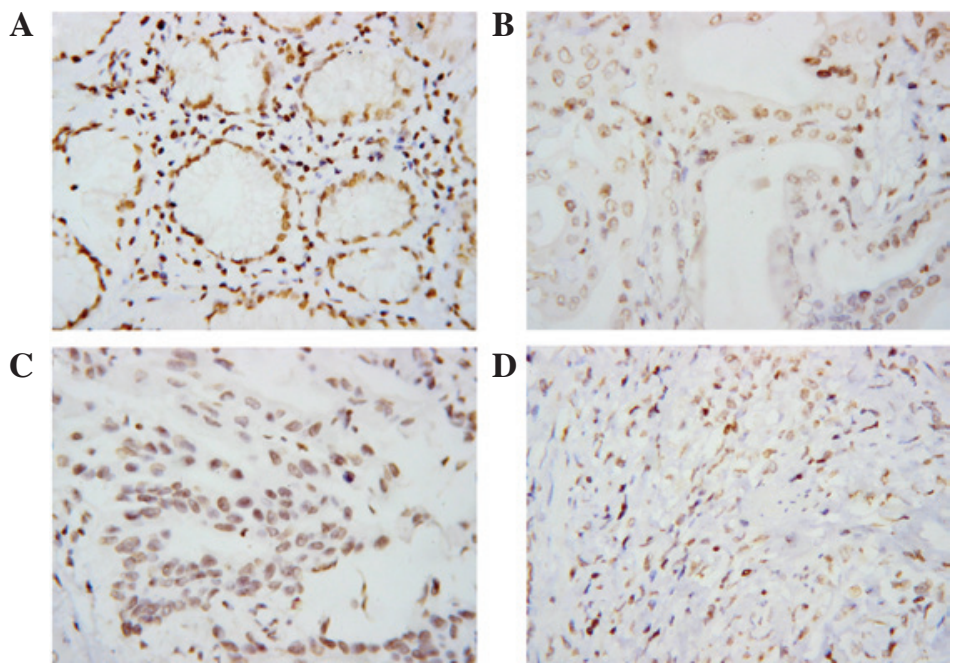

Figure 2. PTEN was mainly distributed in the nucleus. (A) Staining was stronger in normal gastric tissue and (B) well-differentiated gastric cancer and was weaker in (C) moderately and (D) poorly differentiated tumors. Original magnification, x400. PTEN, phosphate and tensin homolog.

Statistical analysis. Statistical analysis was performed using the PASW 18.0 software program for Windows. The results for the correlation between mTOR and PTEN were evaluated using the Chi-squared test. $\mathrm{P}<0.05$ was considered to indicate statistically significant differences.

\section{Results}

Differential expression of mTOR and PTEN in human gastric cancer. The expression levels and cellular distribution of mTOR and PTEN in the 33 specimens of human gastric cancer and 30 normal gastric tissues were examined by immunohistochemical staining. mTOR was distributed mainly in the cytoplasm. Staining was weaker in low-grade tumors. Positive mTOR expression was observed in tumor cells in $51.5 \%$ (17/33) of the gastric cancer patients. By contrast, little or no expression of mTOR was observed in normal gastric tissues (Fig. 1). PTEN was distributed mainly in the nuclei. Staining was stronger in low-grade tumors. Positive PTEN expression was observed in tumor cells in $54.5 \%$ (18/33) of the gastric cancer patients (Fig. 2).

The expression of the mTOR and PTEN antigens was assessed by immunohistochemical staining in sections obtained from 33 gastric cancer patients with various histological diagnoses and pathological staging according to the AJCC handbook (Table II). In early and advanced cases, the respective positive expression rates were $16.7 \%(1 / 6)$ and $59.3 \%(16 / 27)$ for mTOR and $100.0 \%(6 / 6)$ and $44.4 \%(12 / 27)$ for PTEN. In well- and moderately differentiated tissues and in poorly differentiated specimens, the respective positive expression rates were $26.7 \%(4 / 15)$ and $72.2 \%$ (13/18) for mTOR and $73.3 \%(11 / 15)$ and $38.9 \%$ (7/18) for PTEN. In patients with or without lymph node metastasis, the respective positive expression rates were $81.3 \%(13 / 16)$ and $23.5 \%(4 / 17)$ for mTOR and 18.8\% (3/16) and 88.2\% (15/17) for PTEN. In stage I+II and stage III+IV, the respective positive expression rates were $16.7 \%(3 / 18)$ and $93.3 \%(14 / 15)$ for mTOR and $94.4 \%(17 / 18)$ and $6.7 \%(1 / 15)$ for PTEN. 
Table II. Correlations between mTOR and PTEN expression and clinicopathological characteristics in gastric carcinoma cases.

\begin{tabular}{|c|c|c|c|c|c|c|}
\hline \multirow[b]{2}{*}{ Factors } & \multicolumn{3}{|c|}{ mTOR expression } & \multicolumn{3}{|c|}{ PTEN expression } \\
\hline & Positive $(\%)$ & Negative (\%) & P-value & Positive $(\%)$ & Negative $(\%)$ & P-value \\
\hline Gender & & & 0.849 & & & 0.407 \\
\hline Male & $8(53.3)$ & $7(46.7)$ & & $7(46.7)$ & $8(53.3)$ & \\
\hline Female & $9(50.0)$ & $9(50.0)$ & & $11(61.1)$ & $7(38.9)$ & \\
\hline Age (years) & & & 0.221 & & & 0.009 \\
\hline$<54$ & $10(62.5)$ & $6(37.5)$ & & $5(31.3)$ & $11(68.8)$ & \\
\hline$\geq 54$ & $7(41.2)$ & $10(58.8)$ & & $13(76.5)$ & $4(23.5)$ & \\
\hline Invasive depth & & & 0.085 & & & 0.021 \\
\hline Early stage & $1(16.7)$ & $5(83.3)$ & & $6(100.0)$ & $0(0)$ & \\
\hline Advanced stage & $16(59.3)$ & $11(40.7)$ & & $12(44.4)$ & $15(55.6)$ & \\
\hline Differentiation & & & 0.009 & & & 0.048 \\
\hline Well and moderate & $4(26.7)$ & $11(73.3)$ & & $11(73.3)$ & $4(26.7)$ & \\
\hline Poor & $13(72.2)$ & $5(27.8)$ & & 7 (38.9) & $11(61.1)$ & \\
\hline Lymph node metastasis & & & 0.001 & & & 0.000 \\
\hline Positive & $13(81.3)$ & $3(18.8)$ & & $3(18.8)$ & $13(81.3)$ & \\
\hline Negative & $4(23.5)$ & $13(76.5)$ & & $15(88.2)$ & $2(11.8)$ & \\
\hline Pathological stage & & & 0.000 & & & 0.000 \\
\hline $\mathrm{I}+\mathrm{II}$ & $3(16.7)$ & $15(83.3)$ & & $17(94.4)$ & $1(5.6)$ & \\
\hline III+IV & $14(93.3)$ & $1(6.7)$ & & $1(6.7)$ & $14(93.3)$ & \\
\hline
\end{tabular}

mTOR, mammalian target of rapamycin; PTEN, phosphate and tensin homolog.

Table III. Co-expression of mTOR and PTEN and clinicopathological characteristics.

\begin{tabular}{|c|c|c|c|}
\hline Factors & $\mathrm{mTOR}^{-} / \mathrm{PTEN}^{+}$ & $\mathrm{mTOR}^{+} / \mathrm{PTEN}^{-}$ & P-value \\
\hline \multicolumn{4}{|l|}{ Invasive depth } \\
\hline Early stage & 5 & 0 & 0.041 \\
\hline Advanced stage & 9 & 13 & \\
\hline \multicolumn{4}{|l|}{ Differentiation } \\
\hline Well and moderate & 10 & 3 & 0.012 \\
\hline Poor & 4 & 10 & \\
\hline \multicolumn{4}{|c|}{ Lymph node metastasis } \\
\hline Positive & 2 & 12 & 0.000 \\
\hline Negative & 12 & 1 & \\
\hline \multicolumn{4}{|l|}{ Pathological stage } \\
\hline $\mathrm{I}+\mathrm{II}$ & 14 & 0 & 0.000 \\
\hline III+IV & 0 & 13 & \\
\hline
\end{tabular}

mTOR, mammalian target of rapamycin; PTEN, phosphate and tensin homolog.

The expression of mTOR had a significant positive correlation with differentiation, lymph node metastasis and clinical staging $(\mathrm{P}<0.01)$, but was independent of gender, age and invasive depth $(\mathrm{P}>0.05)$. The expression of PTEN was negatively correlated with invasive depth and differentiation $(\mathrm{P}<0.05)$ and significantly negatively correlated with age, lymph node metastasis and clinical pathological staging $(\mathrm{P}<0.01)$, but was not associated with gender $(\mathrm{P}>0.05)$.
Correlation between mTOR and PTEN in human gastric cancer. When the expression of mTOR was analyzed with regard to PTEN expression, the staining pattern was divided into 4 groups: $\mathrm{mTOR}^{-} / \mathrm{PTEN}^{-}, \mathrm{mTOR}^{+} / \mathrm{PTEN}^{+}, \mathrm{mTOR}^{+} / \mathrm{PTEN}^{-}$ and $\mathrm{mTOR}^{-} / \mathrm{PTEN}^{+}$. A comparison of the $\mathrm{mTOR}^{-} / \mathrm{PTEN}^{+}$and $\mathrm{mTOR}^{+} / \mathrm{PTEN}^{-}$groups (Table III) revealed that that in early gastric cancer, the size of the former group was larger than the that of latter and that the differences between the 2 groups were 
statistically significant with regard to invasive depth $(\mathrm{P}=0.041)$. In well- and moderately differentiated gastric cancer, the $\mathrm{mTOR}^{-} / \mathrm{PTEN}^{+}$group was larger than the $\mathrm{mTOR}^{+} / \mathrm{PTEN}^{-}$group and the difference between the 2 groups was statistically significant with regard to differentiation $(\mathrm{P}=0.012)$. In patients without lymph node metastasis, the $\mathrm{mTOR}^{-} / \mathrm{PTEN}^{+}$group was larger than the $\mathrm{mTOR}^{+} / \mathrm{PTEN}^{-}$group and the difference between the 2 groups was statistically significant with regard to lymph node metastasis $(\mathrm{P}=0.000)$. In stage $\mathrm{I}+\mathrm{II}$, the $\mathrm{mTOR}^{-} / \mathrm{PTEN}^{+}$group was larger than the $\mathrm{mTOR}^{+} / \mathrm{PTEN}^{-}$group and the difference between two groups was statistically significant with regard to pathological stage $(\mathrm{P}=0.000)$.

\section{Discussion}

Previous studies have suggested that the PI3K-AKT-mTOR pathway is frequently activated in various types of cancer and that this pathway is considered to be important for cancer cell survival, proliferation, angiogenesis and resistance to chemotherapy (19-21). However, the activated molecule of the PI3K-AKT-mTOR pathway in gastric cancer has not yet been studied. In the present study, 33 cases of gastric cancer were investigated and statistical analyses were performed concerning the correlation between the clinicopathological parameters in gastric cancer and the immunohistochemical expression levels of mTOR and PTEN. The results indicated that mTOR and PTEN were negatively correlated in the pathogenesis of gastric cancer. The overexpression of mTOR and low expression of PTEN proteins were strongly correlated with the pathological staging. These results suggested that mTOR and PTEN may be clinically useful prognostic markers and may provide additional information for the histological diagnosis and pathological staging of gastric cancer.

Possible correlations between mTOR expression in gastric cancer and pathological parameters were investigated. The present study demonstrated that mTOR was activated in human gastric cancer and was significantly correlated with invasive depth, differentiation and lymph node metastasis, suggesting that the high expression of mTOR contributes to the progression and metastasis of gastric cancer. Positive mTOR expression was detected in tumor cells in 51.5\% (17/33) of gastric cancer patients, while little or no expression was observed in normal gastric tissues. Furthermore, 93.3\% (14/15) of gastric cancer patients had positive expression of mTOR at stage III+IV suggesting that the hyperactivation of mTOR kinase was a late event in the development of gastric cancer. Similarly, the positive expression rate of mTOR was high for those patients with lymph node metastasis $(81.3 \%, 13 / 16)$. mTOR immunoreactivity intensity data revealed that the high expression levels of mTOR significantly increased with the tumor progression.

It has been reported that mTOR is a powerful oncoprotein overexpressed in numerous types of cancer, including hepatocellular carcinoma (22), lung cancer (23), esophageal squamous cell carcinoma (24) and breast cancer $(25,26)$. Furthermore, patients with breast cancer and mTOR overexpression had a risk of recurrence 3 times greater than that of patients without mTOR overexpression $(25,27)$. The mTOR inhibitor everolimus has demonstrated promising clinical efficacy in a phase III randomized and double-blind trial in patients with metastatic renal cell cancer (28). The exact mechanisms for the overexpression of mTOR in carcinoma remain unclear. As a highly conserved, ubiquitously expressed signaling molecule, mTOR is activated downstream of multiple distinct growth factor receptors and is crucial for mediating cell proliferation and survival (29). Several mTOR inhibitors, including rapamycin/sirolimus (Wyeth) and derivatives, such as RAD001/everolimus (Novartis), CCI-779/temsirolimus (Wyeth) and AP23573 (Ariad), are being developed as anti-cancer agents against various types of malignancies (30).

By contrast, PTEN was shown by immunohistochemistry to be expressed in normal gastric tissues and almost all the early gastric cancer cases. The underexpression of PTEN was significantly correlated with invasive depth, differentiation and lymph node metastasis. Approximately one-half of the gastric cancer patients exhibited a loss of PTEN expression. Additionally, the loss of PTEN expression was correlated with invasive depth and differentiation $(\mathrm{P}<0.05)$ and closely correlated with lymph node metastasis and clinical pathological staging $(\mathrm{P}<0.01)$. PTEN immunoreactivity intensity data were also analyzed and the results revealed that the high expression levels of PTEN significantly decreased with the tumor progression.

PTEN, which is located at human chromosome 10q23, has been identified as a tumor suppressor gene and an important negative regulator of the PI3K-AKT-mTOR signaling pathway that promotes cell proliferation and inhibits apoptosis (31). Inactivation of PTEN by mutation, deletion and promoter hypermethylation has been demonstrated in a range of cancer types, including lung, breast, prostate and esophageal carcinomas (31-35). Langlois et al observed that PTEN controls the cellular polarity, establishment of cell-cell junctions, paracellular permeability, migration and tumorigenic potential of human colorectal cancer cells (36). Varied analysis of colorectal carcinomas suggested that the patients without PTEN expression had shorter survival times than the patients with PTEN expression ( $\mathrm{P}=0.003$ ) (37). Abnormal expression of PTEN may predict the metastasis and prognosis of gastric cancer $(21,38,39)$.

We concluded that mTOR facilitated the development of gastric cancer while PTEN, a tumor suppressor gene, was able to inhibit tumor invasion and metastasis. mTOR and PTEN co-regulate the progression of tumors and participate in proliferation, invasion and metastasis in gastric cancer. Bakarakos et al discovered that the loss of PTEN and activation of mTOR was closely correlated with breast cancer (40). In vitro studies suggested that PTEN is capable of inhibiting cell proliferation and promoting apoptosis via inhibition of the activity of the PI3K-Akt-mTOR pathway (41). The combined deletion of PTEN and Lkb1 in the mouse bladder significantly activated the mTOR pathway and increased bladder epithelial cell proliferation and tumorigenesis (42). When we compared the $\mathrm{mTOR}^{-} / \mathrm{PTEN}^{+}$and $\mathrm{mTOR}^{+} / \mathrm{PTEN}^{-}$groups, the differences between them were statistically significant with regard to invasive depth, histological type, lymph node metastasis and pathological stage. Consequently, collaborative detection of mTOR and PTEN expression may be more useful in the diagnosis of gastric cancer.

In summary, upregulated expression of mTOR and downregulated expression of PTEN were involved in carcinogenesis and progression of gastric cancer. A negative correlation between mTOR and PTEN expression implied that their modified expression may be important in the pathogenesis, 
invasion and metastasis of carcinoma tissue. Combined detection of mTOR and PTEN expression may be used to evaluate the degree of malignancy in gastric cancer, which may be a useful marker for the early diagnosis of gastric cancer. Further studies with more patients, including follow-up and different molecular biomarkers in addition to these two molecules, would aid the clarification of the disease pathogenesis and identification of potential therapeutic approaches.

\section{Acknowledgements}

The author Min Li gratefully acknowledges the assistance of his elder sister Li Li for her critical reading of the manuscript before its submission. We also gratefully acknowledge the assistance of Xinyu Qin, Huawen Sun and Lujun Song in the preparation of this study.

\section{References}

1. Washington K: 7th edition of the AJCC cancer staging manual: stomach. Ann Surg Oncol 17: 3077-3079, 2010.

2. Liang S, He L, Zhao X, et al: MicroRNA let-7f inhibits tumor invasion and metastasis by targeting myh9 in human gastric cancer. PloS One 6: e18409, 2011.

3. Roukos DH: Targeting gastric cancer with trastuzumab: new clinical practice and innovative developments to overcome resistance. Ann Surg Oncol 17: 14-17, 2010.

4. Bal N, Kocer NE, Ertorer ME, Canpolat ET and Kayaselcuk F: Maspin, E-selectin, and P-selectin expressions in papillary thyroid carcinomas and their correlation with prognostic parameters. Pathol Res Pract 204: 743-750, 2008.

5. Chen X, Liao J, Lu Y, Duan X and Sun W: Activation of the PI3K Akt pathway mediates bone morphogenetic protein 2-induced invasion of pancreatic cancer cells Panc-1. Pathol Oncol Res 17: 257-261, 2011.

6. Xu Z, Zhang Y, Jiang J, et al: Epidermal growth factor induces HCCR expression via PI3K/Akt/mTOR signaling in PANC-1 pancreatic cancer cells. BMC Cancer 10: 161, 2010.

7. Bhatt AP, Bhende PM, Sin SH, Roy D, Dittmer DP and Damania B: Dual inhibition of PI3K and mTOR inhibits autocrine and paracrine proliferative loops in PI3K/Akt/mTOR-addicted lymphomas. Blood 115: 4455-4463, 2010.

8. Saji M and Ringel MD: The PI3K-Akt-mTOR pathway in initiation and progression of thyroid tumors. Mol Cell Endocrinol 321: 20-28, 2010.

9. Vignot S, Faivre S, Aguirre D and Raymond E: mTOR-targeted therapy of cancer with rapamycin derivatives. Ann Oncol 16: 525-537, 2005

10. Mahalingam D, Sankhala K, Mita A, Giles FJ and Mita MM: Targeting the mTOR pathway using deforolimus in cancer therapy. Future Oncol 5: 291-303, 2009.

11. Ching CB and Hansel DE: Expanding therapeutic targets in bladder cancer: the PI3K/Akt/mTOR pathway. Lab Invest 90: 1406-1414, 2010.

12. Carew JS, Kelly KR and Nawrocki ST: Mechanisms of mTOR inhibitor resistance in cancer therapy. Target Oncol 6: 17-27, 2011.

13. Yoshimi A, Goyama S, Watanabe-Okochi N, et al: Evil represses PTEN expression and activates PI3K/AKT/mTOR via interactions with polycomb proteins. Blood 117: 3617-3628, 2011.

14. Mireuta M, Darnel A and Pollak M: IGFBP-2 expression in MCF-7 cells is regulated by the PI3K/AKT/mTOR pathway through Sp1-induced increase in transcription. Growth Factors 28: 243-255, 2010

15. Liu C, Wu JL, Xu K, et al: Neuroprotection by baicalein in ischemic brain injury involves PTEN/AKT pathway. J Neurochem 112: 1500-1512, 2010.

16. Hamilton SR and Aaltonen LA (eds): Pathology and Genetics of Tumors of the Digestive System. WHO Press, Geneva, 2000.

17. Edge SB, Byrd DR, Compton CC, Fritz AG, Greene F and Trotti A (eds): AJCC cancer staging manual. 7th edition Springer, New York, 2010.

18. He X, Wei Q, Zhang X, et al: Immunohistochemical expression of CXCR4 in thyroid carcinomas and thyroid benign lesions. Pathol Res Pract 206: 712-715, 2010.
19. Niu HY, Wang JH, Li H and He P: Rapamycin potentiates cytotoxicity by docetaxel possibly through downregulation of Survivin in lung cancer cells. J Exp Clin Canc Res 30: 28, 2011.

20. Chen M, Gu J, Delclos GL, et al: Genetic variations of the PI3KAKT-mTOR pathway and clinical outcome in muscle invasive and metastatic bladder cancer patients. Carcinogenesis 31: 1387-1391, 2010.

21. Li M, Song L and Qin X: Glycan changes: cancer metastasis and anti-cancer vaccines. J Biosci 35: 665-673, 2010.

22. Sahin F, Kannangai R, Adegbola O, Wang JZ, Su G and Torbenson M: mTOR and P70 S6 kinase expression in primary liver neoplasms. Clin Cancer Res 10: 8421-8425, 2004.

23. Schmid K, Bago-Horvath Z, Berger W, et al: Dual inhibition of EGFR and mTOR pathways in small cell lung cancer. Br J Cancer 103: 622-628, 2010.

24. Hirashima K, Baba Y, Watanabe M, et al: Phosphorylated mTOR expression is associated with poor prognosis for patients with esophageal squamous cell carcinoma. Ann Surg Oncol 17: 2486-2493, 2010

25. Yu G, Wang J, Chen Y, et al: Overexpression of phosphorylated mammalian target of rapamycin predicts lymph node metastasis and prognosis of Chinese patients with gastric cancer. Clin Cancer Res 15: 1821-1829, 2009.

26. An J, Jeong H,Lee Y, Woo SU, Seo JH and Kim A: Phosphorylated Akt and phosphorylated mTOR expression in breast invasive carcinomas: analysis of 530 cases. J Breast Cancer 13: 337-348, 2010.

27. Bose S, Chandran S, Mirocha JM and Bose N: The Akt pathway in human breast cancer: a tissue-array-based analysis. Mod Pathol 19: 238-245, 2006.

28. Motzer RJ, Escudier B, Oudard S, et al: Efficacy of everolimus in advanced renal cell carcinoma: a double-blind, randomised, placebo-controlled phase III trial. Lancet 372: 449-456, 2008.

29. Marinov M, Ziogas A, Pardo OE, et al: AKT/mTOR pathway activation and BCL-2 family proteins modulate the sensitivity of human small cell lung cancer cells to RAD001. Clin Cancer Res 15: 1277-1287, 2009.

30. Tam KH, Yang ZF, Lau CK, Lam CT, Pang RW and Poon RT: Inhibition of mTOR enhances chemosensitivity in hepatocellular carcinoma. Cancer Lett 273: 201-209, 2009.

31. Jang KS, Song YS, Jang SH, et al: Clinicopathological significance of nuclear PTEN expression in colorectal adenocarcinoma. Histopathology 56: 229-239, 2010.

32. Andjelkovic T, Bankovic J, Stojsic J, et al: Coalterations of p53 and PTEN tumor suppressor genes in non-small cell lung carcinoma patients. Transl Res 157: 19-28, 2011.

33. Gonzalez-Angulo AM, Ferrer-Lozano J, Stemke-Hale K, et al: PI3K pathway mutations and PTEN levels in primary and metastatic breast cancer. Mol Cancer Ther 10: 1093-1101, 2011.

34. Mulholland DJ, Tran LM, Li Y, et al: Cell autonomous role of PTEN in regulating castration-resistant prostate cancer growth. Cancer Cell 19: 792-804, 2011.

35. Hou G, Lu Z, Liu M, Liu H and Xue L: Mutational analysis of the PTEN gene and its effects in esophageal squamous cell carcinoma. Dig Dis Sci 56: 1315-1322, 2011.

36. Langlois MJ, Bergeron S, Bernatchez G, et al: The PTEN phosphatase controls intestinal epithelial cell polarity and barrier function: role in colorectal cancer progression. PloS One 5: e15742, 2010

37. Li XH, Zheng HC, Takahashi H, Masuda S, Yang XH and Takano Y: PTEN expression and mutation in colorectal carcinomas. Oncol Rep 22: 757-764, 2009.

38. Guo CY, Xu XF, Wu JY and Liu SF: PCR-SSCP-DNA sequencing method in detecting PTEN gene mutation and its significance in human gastric cancer. World J Gastroenterol 14: 3804-3811, 2008.

39. Li M, Song L, Gao X, Chang W and Qin X: Toll-like receptor 4 on islet $\beta$ cells senses expression changes in high-mobility group box 1 and contributes to the initiation of type 1 diabetes. Exp Mol Med 44: 260-267, 2012.

40. Bakarakos P, Theohari I, Nomikos A, et al: Immunohistochemical study of PTEN and phosphorylated mTOR proteins in familial and sporadic invasive breast carcinomas. Histopathology 56: 876-882, 2010.

41. Cheng ZY, Guo XL, Yang XY, et al: PTEN and rapamycin inhibiting the growth of K562 cells through regulating mTOR signaling pathway. J Exp Clin Cancer Res 27: 87, 2008.

42. Shorning BY, Griffiths D and Clarke AR: Lkb1 and Pten synergise to suppress mTOR-mediated tumorigenesis and epithelial-mesenchymal transition in the mouse bladder. PloS One 6: e16209, 2011. 